\title{
Effect of Local Periodic Disturbance on Mixing Layer Downstream of Two-Dimensional Jet
}

\section{(Spatial Structure and Quantitative Representation of Laminar-Turbulent Transition Process)*}

\author{
Masashi ICHIMIYA**, Shinya KAMADA***, Akiyoshi OKAJIMA*** \\ and Takayuki OSAKI**** \\ **Institute of Technology and Science, The University of Tokushima, \\ 2-1 Minami-Josanjima-cho, Tokushima-shi, Tokushima, 770-8506 Japan \\ E-mail: ichimiya@tokushima-u.ac.jp \\ ***Graduate School, The University of Tokushima \\ ****Institute of Technology and Science, The University of Tokushima
}

\begin{abstract}
The laminar-turbulent transition of a mixing layer induced by oscillating flat plates at an exit of a two-dimensional nozzle was experimentally investigated. The mixing layer was formed between the jet, which issued from the nozzle and the surrounding quiescent fluid. The plates oscillated vertically in relation to the mean flow. The oscillation frequency was two orders of magnitude smaller than the fundamental frequency of the velocity fluctuation. Mean and fluctuating velocity components in the streamwise and normal directions were measured by hot-wire anemometers. In the oscillating state, the same phenomenon as in the natural transition process appeared more upstream. In the early stage within the nonlinear region, the growth of fluctuating velocity weakened and the Reynolds shear stress component decreased. The decrease in their production rate due to the expansion of the mixing layer contributed to the weakening and decrease. The probability of the streamwise and normal fluctuating velocity components taking the same sign increased or decreased in accordance with the increase and decrease of the Reynolds shear stress component. The randomness factor, which had been proposed by Sato, appeared to be a reasonable indicator of the present transition process, especially in the process in which the periodic velocity fluctuation became irregular. However, this factor certainly indicated the same value at two streamwise positions.
\end{abstract}

Key words: Mixing Layer, Transition, Turbulence, Jet, Shear Flow, Oscillating Plate, Periodic Disturbance, Nonlinear Region, Randomness Factor

\section{Introduction}

The laminar-turbulent transition phenomenon has been investigated for many years, because it occurs very often in the natural world and in industrial machinery. A wake of turbine cascades in industrial machines ${ }^{(1)}$ is an example of the laminar-turbulent transition in free shear flow without constraint by a solid wall. As another example of free shear flow, in the early stage of a jet development, both the potential core in which the velocity is constant, and the mixing layer in which the velocity has a gradient, are formed. The 
laminar-turbulent transition process in the mixing layer is classified into three regions. First, the linear region appears where disturbances grow exponentially and a periodic fluctuation, i.e., a fundamental wave, is observed ${ }^{(2)}$. Next, the nonlinear region appears where the harmonic and subharmonic waves of the fundamental wave are observed. At last, the irregular region appears where an irregular fluctuation dominates.

In the case of a boundary layer, during the last stage of the laminar-turbulent transition process, isolated turbulent patches, "turbulent spots," are formed and develop downstream. Therefore, the intermittency factor, which is the time ratio of the turbulence, has been used as the indicator of the transition process. It is known to increase monotonically downstream. On the other hand, regarding the transition process of the free shear flow as in the present investigation, Sato et al. proposed the randomness factor as the indicator based on the variation of the power spectrum density of the velocity fluctuation, although it seems not to have been used until recently ${ }^{(3)(4)}$.

In many studies concerning the transition in the mixing layer and the jet before an establishment of self-similarity, the effects of forced disturbance have been investigated. Representative studies are mentioned in the previous paper ${ }^{(5)}$. As one example of a forced disturbance, a periodic disturbance has been introduced, mainly as a frequency equal to the fundamental frequency of velocity fluctuation in the linear region of the natural transition process. Though the mixing layer is unstable under disturbances with a low wave number, i.e., a long wavelength in contrast with the boundary layer ${ }^{(6)}$, the transition process with a periodic disturbance with a long wavelength has not been investigated.

The present study deals with the transition process in a mixing layer formed between a jet just after a two-dimensional nozzle and the surrounding quiescent fluid. The fence-type flat plates oscillated and protruded vertically in relation to the jet with low frequency, which is two orders of magnitude lower than the fundamental frequency. In the previous paper, protrusion of the plate was so small that a disturbance by the protrusion itself was not created $^{(5)}$. The streamwise velocity component and development of the mixing layer were measured. From these measurements, the relation between the velocity fluctuation and the energy production, dissipation and convection rates of the perturbation was examined. The transition process downstream of the nonlinear region and the extension of the mixing layer in the normal direction, however, have not been elucidated.

The present paper examines the extension of the transition process in the normal direction at first; then the decrease of the disturbance growth in the nonlinear region is examined. Finally, the advantage and disadvantage of the randomness factor as an indicator of the transition process in the mixing layer are examined.

\section{Nomenclature}

$f \quad$ : frequency of velocity fluctuation

$f_{0} \quad$ : fundamental frequency of periodic velocity fluctuation

$f_{e} \quad$ : oscillation frequency of oscillating plates $=5 \mathrm{~Hz}$

$f_{p} \quad$ : preferred frequency of periodic velocity fluctuation

$h \quad$ : nozzle exit height $=10 \mathrm{~mm}$

$P(f)$ : power spectrum density of streamwise fluctuating velocity at frequency $f$ $p\left(u / u^{\prime}, v / v^{\prime}\right)$ : joint probability density function between $u$ and $v$

$p_{u, v, i}$ : probability in which value of $u$ and $v$ is included within $i$-th quadrant

$U, u:$ mean and fluctuating velocity component in streamwise direction

$U_{0} \quad$ : velocity at $x=5 \mathrm{~mm}, y=0$

$U_{m} \quad$ : local velocity on the centerline

$V, v:$ mean and fluctuating velocity component in normal direction

$u^{\prime}, v^{\prime}:$ root mean square value of $u$ and $v$, respectively

$x, y$ : coordinate system 


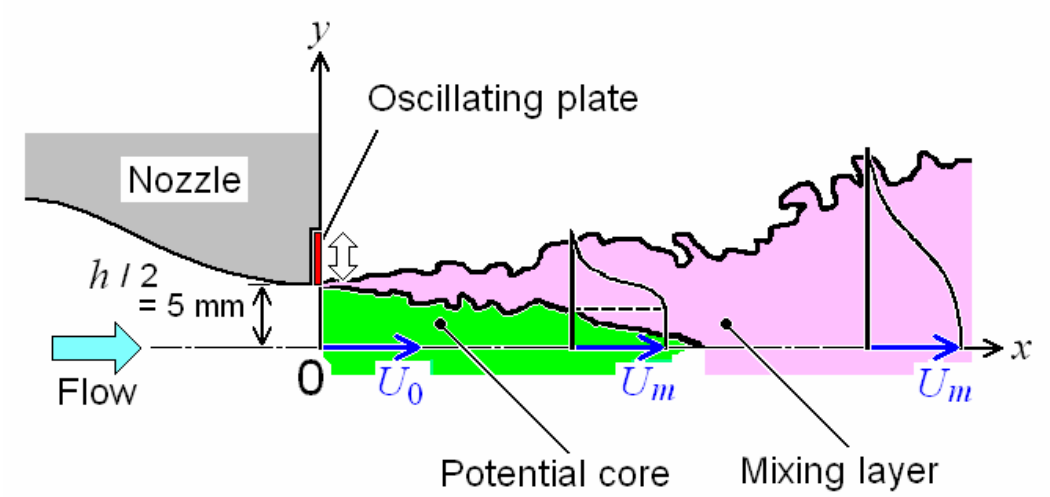

Fig. 1 Schematic diagram of two-dimensional mixing layer and coordinate system.

$z \quad$ : randomness factor

* $\quad$ : time average of quantity $*$

\section{Experimental Apparatus and Methods}

The experimental apparatus is the same one used in a previous paper ${ }^{(5)}$. The mixing layer is formed just downstream of a two-dimensional nozzle with an aspect ratio of 31 (310 $\mathrm{mm}$ in width and $10 \mathrm{~mm}$ in height, $h$ ). For the convenience of the reader, the upper half of the flow field and coordinate system are reproduced in Fig. 1. Two types of experiments were performed. In one, the oscillating plates which were installed at the exit of the nozzle remained stationary so that the plates would not narrow the nozzle exit section (stationary state). In another, the plates oscillated at a frequency of $5 \mathrm{~Hz}$ and an amplitude of $0.25 \mathrm{~mm}$ (oscillating state). In both cases, the Reynolds number was 5000 based on the nozzle exit velocity without oscillation, $U_{0} \simeq 7.5 \mathrm{~m} / \mathrm{s}$, and the nozzle exit height, $h$. X-shaped hot-wire probes with two tungsten sensing elements, each $5 \mu \mathrm{m}$ in diameter and $1 \mathrm{~mm}$ in length, were used for the measurements. Output voltage was sampled at a sampling frequency of 5 $\mathrm{kHz}$ for about 52 seconds. This interval corresponds to about 260 oscillations in the oscillating state. The measurements were conducted in a range of $y \geqq 0$. Results are shown here in the range $x / h \leqq 25$ (stationary state) and $x / h \leqq 20$ (oscillating state), where the mean velocity profiles had become identical.

\section{Results and Discussion}

\subsection{Spatial Distribution of Fluctuation in Linear Region}

From the exponential increase of the streamwise fluctuating velocity, the linear region was estimated as $x / h \leqq 3$ or $\leqq 1.5$ in the stationary or oscillating regions, respectively ${ }^{(5)}$. In the nonlinear region downstream, the increasing rate of fluctuating velocity decreased. The distributions in the normal direction of the periodic and irregular fluctuations within linear and nonlinear regions will be observed in this section.

Figures 2 and 3 show instantaneous velocity signals and the power spectrum density of the streamwise fluctuating velocity at the terminal of the linear region, $x / h=3$ and 1.5 , in the stationary and oscillating states, respectively. The figures are shown at three normal positions. They are $(a)$, on the centerline, $y /(h / 2)=0 ;(b)$, at the edge of the potential core region, $y /(h / 2)=0.60$ and 0.68 in the stationary and oscillating states, respectively; $(c)$, and in the position of maximum streamwise fluctuating velocity, at $y /(h / 2)=1.0$ and 0.95 , in the stationary and oscillating states, respectively. The region of the abscissa in the instantaneous velocity signal was 0.2 seconds, which is the same as one period of the plate oscillation in the oscillating state. The integration of the power spectrum density, $P(f)$ within the whole 

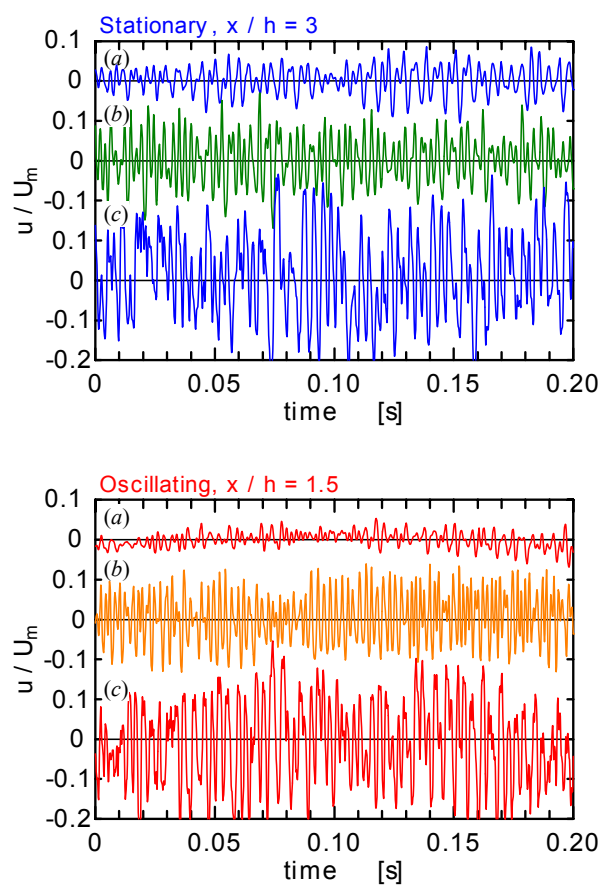

Fig. 2 Fluctuating velocity signals in the streamwise component: stationary, $x / h=3$; oscillating, $x / h=1.5$.
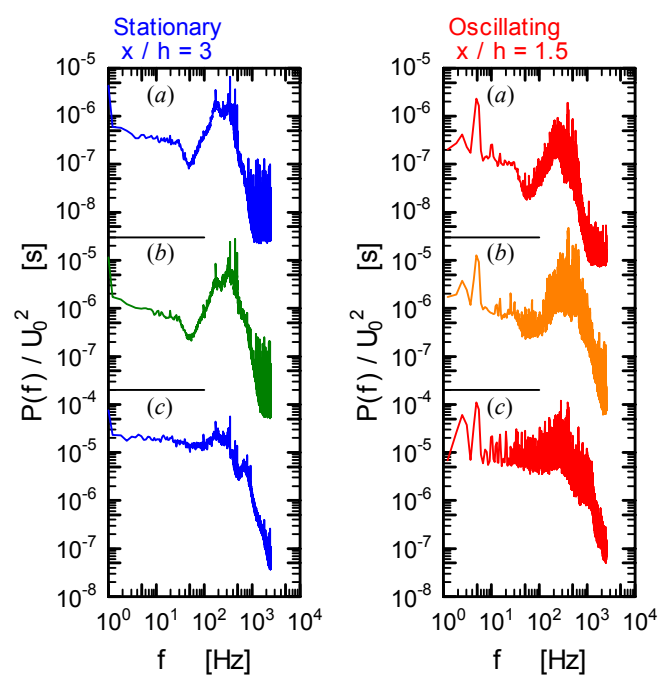

Fig. 3 Power spectrum density of the streamwise fluctuating velocity: stationary, $x / h=3$; oscillating, $x / h=1.5$.

frequency region became $\overline{u^{2}}$. Here, the density was normalized by the square of the centerline velocity, $U_{0}$. In the stationary state in the whole $y$ position, periodic fundamental waves with frequencies of approximately $500 \mathrm{~Hz}$, and subharmonic waves with frequencies of approximately 200 and $300 \mathrm{~Hz}$, can both be seen in the velocity signal and the power spectrum density. Additionally, in Figs. 2(c) and 3(c), periodic harmonic wave whose frequency is approximately $1000 \mathrm{~Hz}$ can be seen. Many peaks which can be seen in Figs. $3(a)$ and $3(b)$ in the stationary state are small peaks exaggerated by the log scale, though the values are very small due to the small fluctuating velocity around these stations.

In the oscillating state in the whole $y$ position, a periodic wave whose frequency is 5 $\mathrm{Hz}$, corresponding to the plate oscillation frequency, can be seen. The periodic wave whose frequency is approximately $400 \mathrm{~Hz}$ is superposed on it. In Figs. $2(b)$ and $3(b)$, the fundamental wave dominates. Additionally, in Figs. 2(a) and 3(a), the subharmonic wave can be seen, and in Figs. 2(c) and 3(c), the harmonic wave can be seen. In this way, in the oscillating state, besides the $5 \mathrm{~Hz}$-wave, the fundamental, subharmonic and harmonic waves can be seen as during their stationary state.

In this way, the dominant frequency varies with the normal position, $y$, and therefore, the distribution of the dominant frequency in the normal direction will be examined in detail. Figure 4 shows contour maps of the power spectrum density of the fluctuating velocity at subharmonic, fundamental and harmonic frequencies. Here, from the peak frequency in the whole measurement region, the fundamental frequency, $f_{0}$, was determined as $485 \mathrm{~Hz}$ or $408 \mathrm{~Hz}$ in the stationary or oscillating states, respectively. The contour maps were drawn until a position within the nonlinear region. Just after the nozzle exit, there were positions where a large value of $y$ was not measured. In addition, the maps were drawn from $x / h=0$ for convenience, though the measurements were performed in the region of 0.5 $\leqq x / h$. In Fig. 4 , in the region where the mean velocity gradient $-\partial U / \partial y$ exists, white lines were drawn where $U / U_{m}$ equals 0.99 and 0.05 . The velocity gradient was smaller on the outside, therefore, the criterion in the outside region was selected not as 0.01 but as 0.05 . Only in the map of the harmonic frequency, Fig. 4(c), were the values one order of magnitude smaller. 


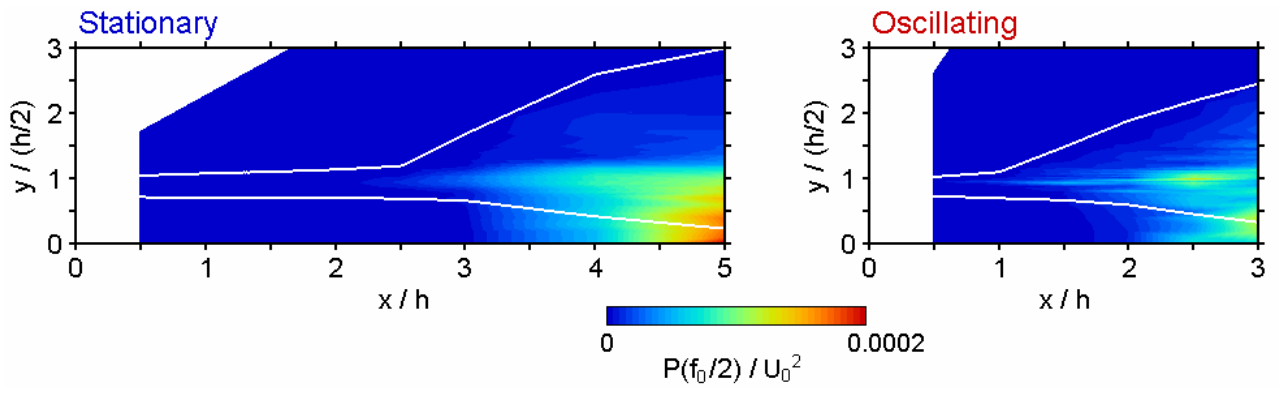

(a)

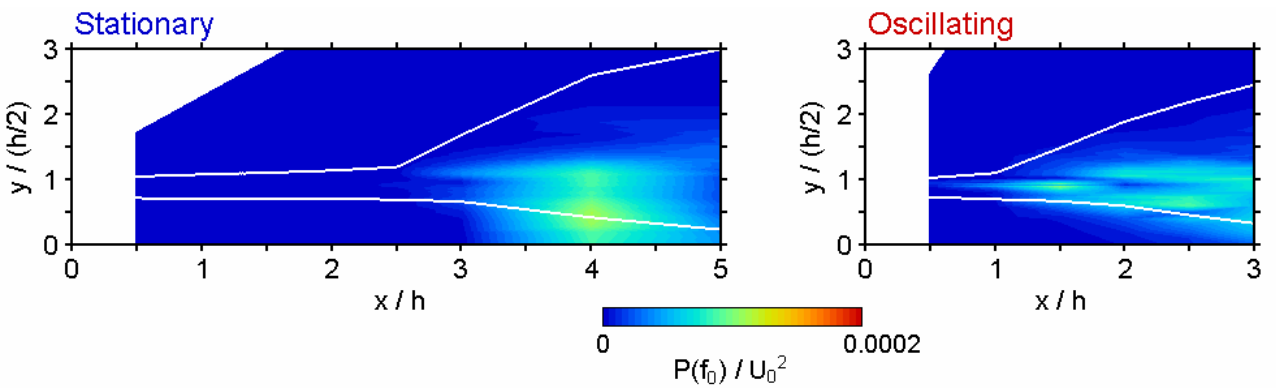

(b)

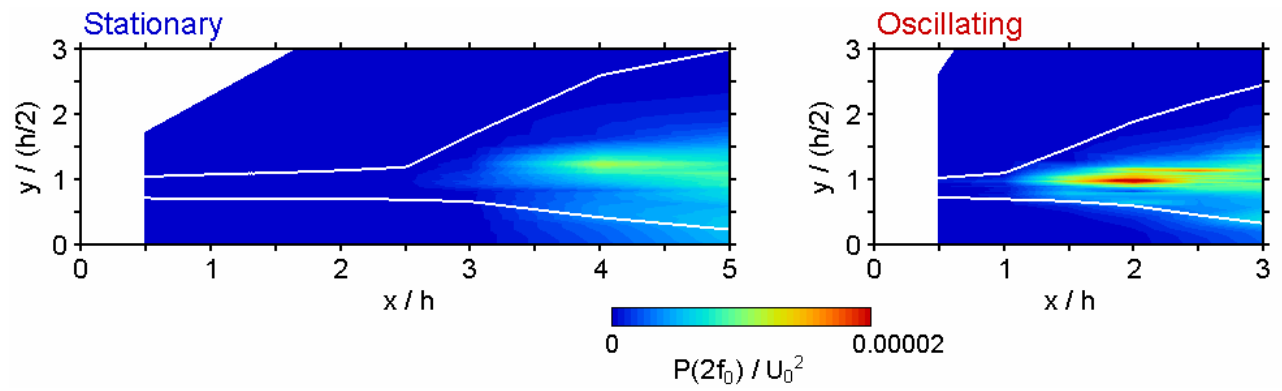

(c)

Fig. 4 Isocontour maps of power spectrum density of fluctuating velocity in the streamwise component at subharmonic, fundamental and harmonic frequencies: $(a)$, subharmonic; $(b)$, fundamental; and $(c)$, harmonic frequencies.

In the stationary state at around $x / h=3$, the terminal of the linear region and within the region where $-\partial U / \partial y$ exists, positions where the power spectrum density at the fundamental frequency has a relatively large value, $y /(h / 2)=0.9$ and 1.1 , and positions where it has a relatively small value, $y /(h / 2)=1.0$, exist side by side as shown in Fig. $4(b)$. On the contrary, the power spectrum density at the subharmonic frequency is relatively large and small, at around $y /(h / 2)=1.0$ and 1.1, respectively in Fig. 4(a). The fact that the maximum and minimum positions of the power spectrum density at fundamental and subharmonic frequencies are opposite each other can be seen in the case of sonic disturbance in Sato ${ }^{(7)}$. Hsiao-Huang examined distributions of power spectrum density at the fundamental and subharmonic frequencies, not in the normal direction but in the streamwise direction ${ }^{(8)}$. They explained the appearance of the subharmonic wave as the coalescence of the vortices of the fundamental frequency. In addition, they measured until the preferred frequency remained as the consecutive coalescence process. The preferred frequency in the present investigation will be described in section 3.3 .

The nonlinear region of stationary state, $3 \leqq x / h$, the region where - $\partial U / \partial y$ exists, extends and approaches the centerline. Accordingly, the power spectrum density at the fundamental frequency reaches a maximum at $x / h=4, y /(h / 2)=0.5$. It is well-known that in the nonlinear region, subharmonic and harmonic waves appear ${ }^{(2)}$. In the present investigation, the spectrum of the fundamental frequency in Fig. 4(b) becomes maximum at 
around $x / h=4$ in the nonlinear region. On the contrary, the subharmonic frequency in Fig. $4(a)$ becomes maximum at around $x / h=5$, well within the nonlinear region. The harmonic frequency in Fig. $4(c)$ also becomes maximum at $x / h=4, y /(h / 2)=1.3$ in the nonlinear region, though it is one order of magnitude smaller than that at fundamental and subharmonic frequencies.

In the oscillating state in the linear region, $x / h \leqq 1.5$, the spectrum of the fundamental frequency in Fig. $4(b)$ becomes minimum at around $y /(h / 2)=1.0$ as well as in the stationary state. In addition, in the larger or smaller $y$-positions of the minimum position, the values show maximum as well as in the stationary state. The streamwise station, however, where the spectrum of the fundamental frequency becomes maximum, is around $x / h=1.5$, where the terminal of the linear region, in opposition to the stationary state. The fact that the maximum and minimum positions of the power spectrum density at fundamental and subharmonic frequencies are opposite each other also holds as in the stationary state. The spectrum in the subharmonic frequency becomes the largest in the nonlinear region as well as in the stationary state. The spectrum in the harmonic frequency becomes largest just after the start of the nonlinear region. In this way, in the oscillating state, respective events occur earlier than in the stationary state. Due to the small amplitude and low frequency of the disturbance which is induced by the plate oscillation, however, the difference between the stationary and oscillating states is nothing but the shift of maximum stations of spectrum in the fundamental wave in the upstream direction.

The normal position where the minimum value of spectrum in the fundamental wave exists is $y /(h / 2) \simeq 1.0$ as seen in Fig. $4(b)$. The phase reverses between regions $y /(h / 2)<1.0$ and $1.0<y /(h / 2)$ in the present stationary state. In the forced disturbance with the sound wave in a two-dimensional jet, Sato ${ }^{(9)}$ confirmed that at the minimum position of the spectrum in the forced frequency, the phase of the periodic motion with the frequency reverses. The present investigation found that in the oscillating state, phase reversal with respect to the oscillating frequency was confirmed.

\subsection{Velocity Fluctuation in Nonlinear Region}

In the nonlinear region, the growth of velocity fluctuation is weaker than in the linear region $^{(2)(5)}$. In this section, the nature of velocity fluctuation will be described. Figure 5 shows the streamwise variation of the maximum values of the root mean square value of fluctuating velocity components in the streamwise and normal direction. The maximum values are determined from the distribution in the $y$-direction. In the previous paper, to define the linear region, streamwise distributions of both maximum values were drawn just after the nozzle. Here, they are drawn in linear scale until well downstream. The defined nonlinear regions, $3 \leqq x / h \leqq 15$ or $1.5 \leqq x / h \leqq 10$ in the stationary or oscillating states, respectively, are also shown in Fig. 5. In both cases, the increasing rate of the maximum values weakens in the nonlinear region, and then the values decrease. The decrease is due to the velocity decrease in the whole $y$-position according to the expansion of the mixing layer in the $y$-direction. In fact, when normalized by the local centerline velocity, $U_{m}$, the maximum values are almost constant at $10 \leqq x / h$. In this way, when the

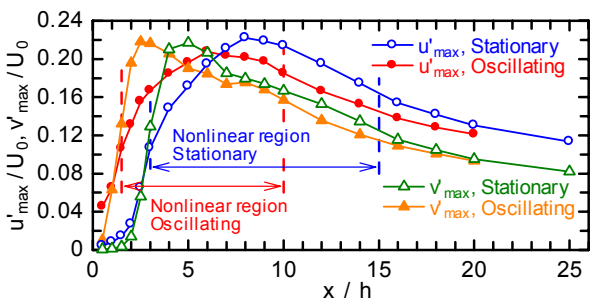

Fig. 5 Streamwise variation of maximum fluctuating velocities.

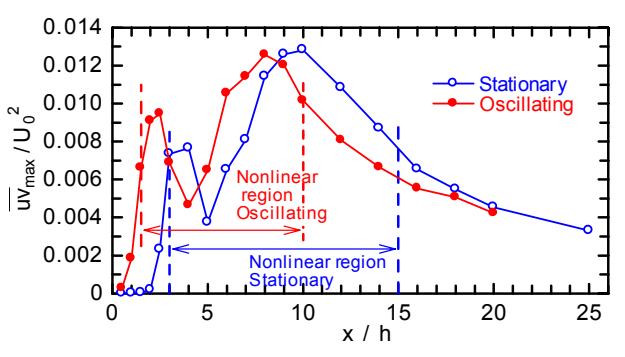

Fig. 6 Streamwise variation of maximum Reynolds shear stress component. 
distribution in the stationary state is shifted upstream, it almost corresponds to the oscillating state. That is, the general shape of distribution does not change due to the small amplitude of the oscillating plate, $0.25 \mathrm{~mm}$. The amplitude did not create a new disturbance by the protrusion itself. The potential core disappears at $x / h \simeq 6$ and 4 in the stationary and oscillating states, respectively.

Figure 6 shows the streamwise variation of the maximum value of the Reynolds stress component. The increasing rate of the maximum value weakens drastically in the nonlinear region, and the maximum value decreases next. The decrease ceases immediately, and increases again in $5 \leqq x / h \leqq 10$ or $4 \leqq x / h \leqq 8$ in the stationary and oscillating states, respectively. In the latter half of the nonlinear region, they decrease as well as $u_{\text {max }}$ and $v_{\text {max }}$. The distribution of the oscillating state is almost the same as the stationary state if it is shifted upstream. The first peak value within the nonlinear region, however, is larger than the stationary state. That is, the plate oscillation disturbance increases the Reynolds shear stress component just after the nozzle exit, though the effect does not continue for long.

Here, the variation of the Reynolds shear stress component shown in Fig. 6 is examined in relation to its production rate. If the boundary layer simplification is introduced, the equation of the Reynolds shear stress component becomes ${ }^{(10)}$,

$$
U \frac{\partial \overline{u v}}{\partial x}+V \frac{\partial \overline{u v}}{\partial y}=-\overline{v^{2}} \frac{\partial U}{\partial y}+\frac{1}{\rho} \overline{p\left(\frac{\partial u}{\partial y}+\frac{\partial v}{\partial x}\right)}-\frac{\partial}{\partial y}\left(\overline{u v^{2}}+\frac{\overline{u p}}{\rho}\right)+v\left(\overline{v \nabla^{2} u}+\overline{u \nabla^{2} v}\right)
$$

The most dominant production rate in Eq. (1) is $-\overline{v^{2}} \partial U / \partial y$. Other production rates based on the velocity gradient in $y$-direction, $-\overline{u v} \partial V / \partial y$, and those based on velocity gradient in the $x$-direction are negligibly small as well, as with Ref. (10). The maximum value of the production rate $-\overline{v^{2}} \partial U / \partial y$ in the distribution in $y$-direction is shown in Fig. 7 . The Reynolds shear stress component becomes maximum at $x / h=4$ and 2.5 in the stationary and oscillating states, respectively, during the early stage in the nonlinear region in Fig. 6. The production rate in Fig. 7 becomes maximum at almost the same stations. Thus, the increase in the linear region and the decrease in the nonlinear region of the Reynolds shear stress component correspond to the variation of its production rates. In this way, oscillation of the plates in the normal direction increases the production rate of the Reynolds shear stress component.

The reason why the production rate decreases in the early stage of the nonlinear region will be examined here. This decrease is caused by the decrease of two factors in the production rate, i. e., the velocity gradient $-\partial U / \partial y$ and turbulent energy $\overline{v^{2}}$. The former decreases immediately and the latter decreases later in the nonlinear region ${ }^{(5)}$. The decrease of the former is due to an expansion of the mixing layer. On the other hand, the decrease of the latter can be explained from the decrease of its production and convection rates, $-\overline{v^{2}} \partial$ $V / \partial y$ and $(U \partial / \partial x+V \partial / \partial y)\left(\overline{v^{2}} / 2\right)$, respectively, as mentioned before. The decrease of the production rate is caused by the decrease of $\overline{v^{2}}$ itself and of a velocity gradient, $-\partial V /$ $\partial y$, i. e., the expansion of the mixing layer. The decrease of the convection rate is mainly caused by the decrease of $\partial \overline{v^{2}} / \partial y$, i. e., the expansion of the mixing layer. In any case, the

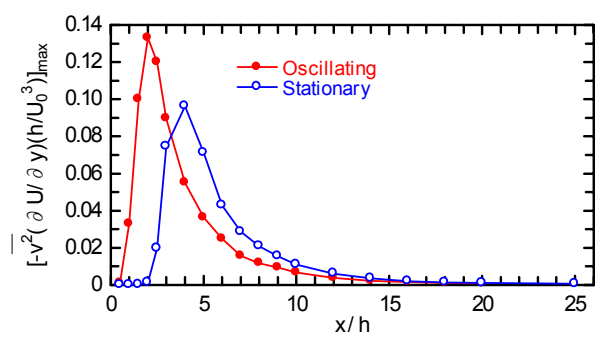

Fig. 7 Streamwise variation of maximum production rates of Reynolds shear stress component. 

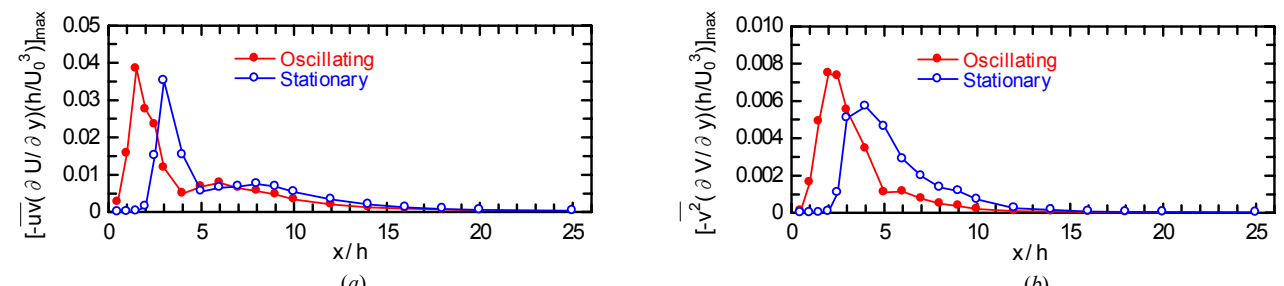

(a)

Fig. 8 Streamwise variation of maximum fluctuating energy production rates in the $(a)$ streamwise and $(b)$ normal components.
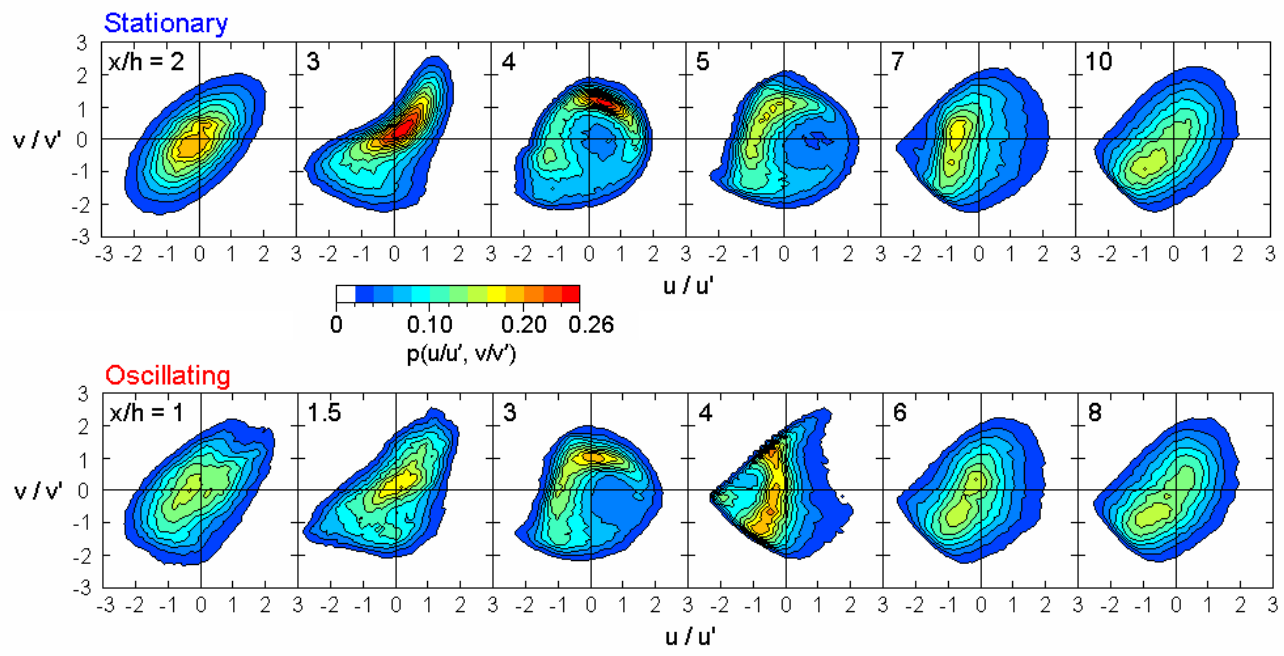

Fig. 9 Isocontour maps of joint probability density function of streamwise and normal fluctuating velocities.

decrease of the Reynolds shear stress component, $\overline{u v}$, is caused by the expansion of the mixing layer.

Next, after $\overline{u v}_{\max }$ decreased, it increased again in $5 \leqq x / h \leqq 10$ and $4 \leqq x / h \leqq 8$ in the stationary and oscillating states, respectively. The increase of $u^{\prime}$ in $x / h \leqq 8$ and $x / h$ $\leqq 6$ in the stationary and oscillating states, respectively, may have contributed to it.

In the previous paper, the distributions in the $y$-direction of the dominant production rate in $\overline{u^{2}}$ and $\overline{v^{2}}$, i. e., $-\overline{u v} \partial U / \partial y$ and $-\overline{v^{2}} \partial V / \partial y$, respectively, are shown in the region of $x / h \leqq 6^{(5)}$. Here, the maximum values in the distribution in the $y$-direction at the respective $x / h$ are shown in Fig. 8 in a streamwise direction. The production rate in $\overline{u^{2}}$ in Fig. 8(a) immediately decreases in the nonlinear region due to the decrease in $\overline{u v}$ itself, and $-\partial U / \partial y$ as mentioned before. The production rate in $\overline{v^{2}}$ in Fig. $8(b)$ also decreases early in the nonlinear region due to the decrease in $\overline{v^{2}}$ itself and $-\partial V / \partial y$. The production rate in $\overline{u^{2}}$ in the oscillating state in Fig. $8(a)$ was almost the same as the rate in the stationary state when it was shifted upstream. On the other hand, when the production rate in the stationary state of $\overline{v^{2}}$ in Fig. $8(b)$ is shifted upstream, and when the peak value is increased, the rate almost corresponds to the one in the oscillating state. Thus, the oscillation of the plates in the normal direction increases the production rate of the fluctuating velocity in the normal direction.

Next, the first decrease and the following increase of the Reynolds shear stress component in the nonlinear region is examined from the aspect of streamwise and normal fluctuating velocity. The contribution of the respective velocity is viewed from a joint probability density function (jpdf) of both directions. Figure 9 shows the contour maps of the jpdf in 6 representative stations. The y-positions in the respective stations are the maximum positions of $\overline{u v}$. The surface integral of the jpdf in the respective figure becomes unity. The maps in the oscillating state almost correspond to those in the stationary state 

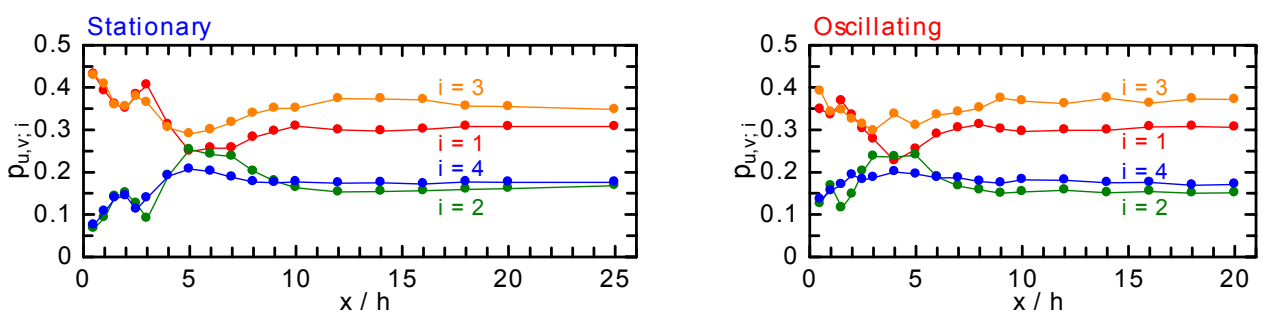

Fig. 10 Probability that the fluctuating velocity takes values of respective quadrant.

when they are shifted upstream. The characteristics of the maps in both states are examined below.

At $x / h=2$ and 1 in the stationary and oscillating states, respectively, where the $\overline{u v}_{\max }$ increases in the linear region, the jpdf distributes widely in the first quadrant where $u \geqq 0$ and $v \geqq 0$, and in the third quadrant where $u \leqq 0, v \leqq 0$. This fact confirms that $\overline{u v}$ takes a positive value. In addition, the jpdf is almost symmetric with the line of $u / u^{\prime}=v / v^{\prime}$.

At $x / h=3$ and 1.5 in the stationary and oscillating states, respectively (the terminal of the linear region), the shape of the jpdf somewhat deforms. Moreover, the area in which the jpdf distributes is small and large in the first and third quadrants, respectively. In the region of $4 \leqq x / h \leqq 5$ and $3 \leqq x / h \leqq 4$ in the stationary and oscillating states, respectively, where the $\overline{u v}_{\max }$ decreases in the nonlinear region, the pdf starts to distribute in the second and fourth quadrant. In the second and fourth quadrants, both fluctuating velocity components contribute $\overline{u v}$ negatively. Thus, the decrease of the $\overline{u v}_{\max }$ in the early stage of the nonlinear region is confirmed.

In the region of $5 \leqq x / h \leqq 10$ and $4 \leqq x / h \leqq 8$ in the stationary and oscillating states, respectively, where the $\overline{u v}_{\max }$ increases again, the distribution of the pdf again becomes symmetric with the line of $u / u^{\prime}=v / v^{\prime}$. At $x / h=10$ and 8 in the stationary and oscillating states, respectively, the jpdf once again distributes mainly in the first and third quadrants.

To express the distribution of the jpdf in four quadrants quantitatively, the area integration of the jpdf in the respective quadrant was obtained, i.e., the probability that both fluctuating velocities exist in the $i$-th quadrant, $p_{u, v, i}$. Figure 10 shows streamwise variations of the four probabilities. During the $\overline{u v}_{\max }$ increases in the linear region, the $p_{u, v, 1}$ and $p_{u, v ; 3}$ decrease and $p_{u, v, 2}$ and $p_{u, v ; 4}$ increase. In the nonlinear region and during the decrease of $\overline{u v}_{\max }$, this tendency continues. At $x / h=5$ and 4 in the stationary and oscillating states, respectively, where $\overline{u v}_{\max }$ takes minimum, the $p_{u, v, 1}$ and $p_{u, v ; 3}$ take minimum correspondingly. On the other hand, the $p_{u, v, 2}$ and $p_{u, v ; 4}$ take maximum. Downstream, in the region of $5 \leqq$ $x / h \leqq 10$ and $4 \leqq x / h \leqq 8$ in the stationary and oscillating states, respectively, where $\overline{u v}_{\max }$ increases again, the $p_{u, v ; 1}$ and $p_{u, v ; 3}$ increase and $p_{u, v, 2}$ and $p_{u, v ; 4}$ decrease. In the region of $10 \leqq x / h$ and $8 \leqq x / h$ in the stationary and oscillating states, respectively, the probabilities are almost constant and the same as the value at the station of $x / h \simeq 16$ and 10 in the stationary and oscillating states, respectively, where the turbulent transition is completed. The constant values of the sum of the $p_{u, v, 1}$ and $p_{u, v ; 3}$ that contribute positively to $\overline{u v}$ are approximately 0.66 and 0.68 in the stationary and oscillating states, respectively. On the other hand, the sum of the $p_{u, v ; 2}$ and $p_{u, v ; 4}$ that contribute positively to $\overline{u v}$ are approximately 0.34 and 0.32 in the stationary and oscillating states, respectively. In this way, the constant values between the stationary and oscillating states in their respective quadrant are almost small. Thus, the present disturbance does not affect the characteristics of the turbulent transition process, though it accelerates the transition. In this way, through the decrease and re-increase processes of the $\overline{u v}_{\max }$, the probabilities change. The tendency to change in the nonlinear region is divided into three regions; the region during the decrease of $\overline{u v}_{\text {max }}$, the region during the re-increase of $\overline{u v}_{\text {max }}$, and the constant-probability region. 

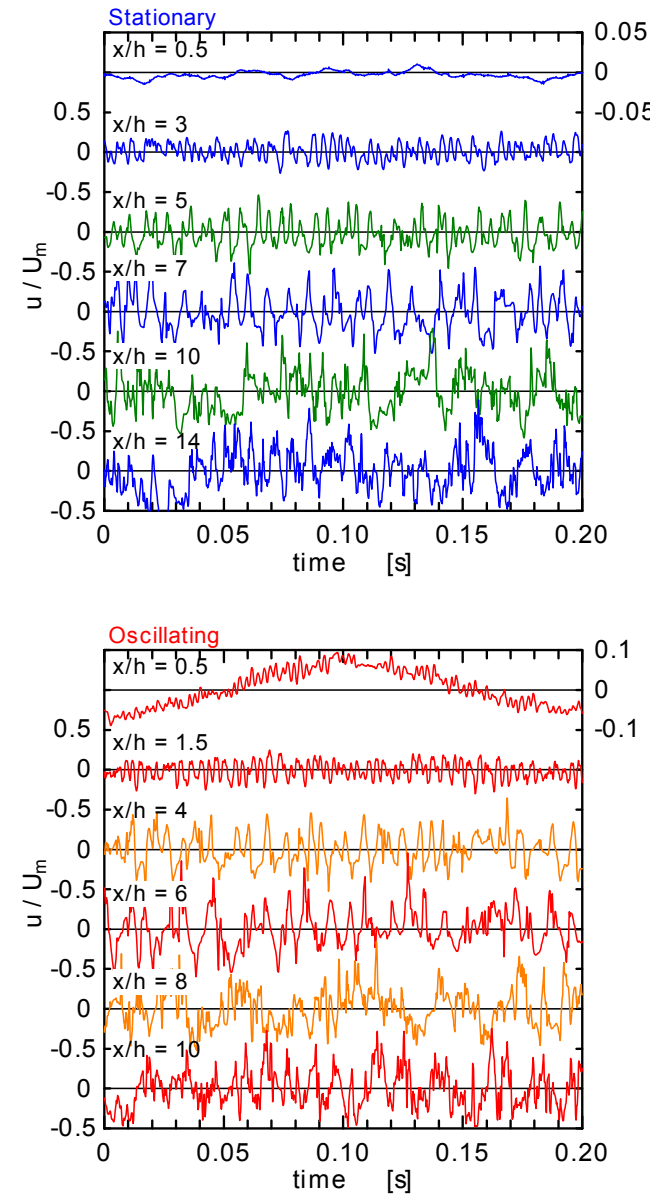

Fig. 11 Fluctuating velocity signals in the streamwise component.
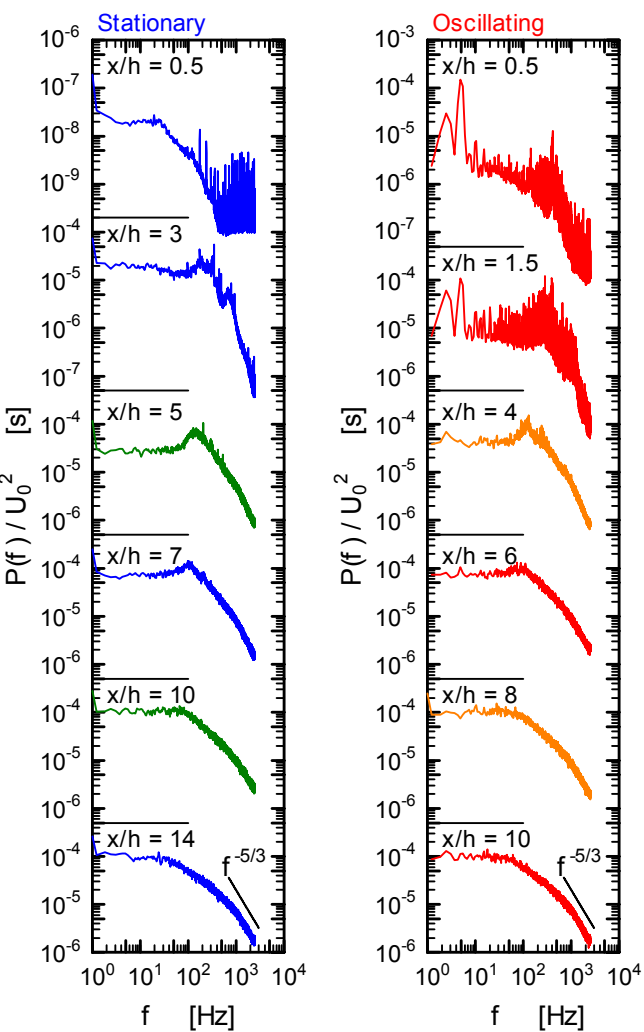

Fig. 12 Power spectrum density of the streamwise fluctuating velocity.

\subsection{Quantitative Representation of Progress of Laminar-Turbulent Transition}

In this section, the laminar-turbulent transition process of the mixing layer throughout the linear, nonlinear and random regions will be quantitatively presented. First of all, the general characteristic of the transition in the present experiment is described.

Figures 11 and 12 show instantaneous velocity signals and power spectrum densities of the streamwise fluctuating velocity at six streamwise stations. They are shown at the normal position, $y$, where $u$ ' takes maximum value at the respective $x$. The velocity signals are shown during 0.2 seconds, i.e., one period. In Fig. 11, the magnitude of the ordinate at $x / h=$ 0.5 in the stationary and oscillating states is expanded more than the others. In what follows, the characteristics of respective stations are examined from both figures.

In the stationary state, few fluctuations are observed at $x / h=0.5$. The power spectrum density is small due to normalization by $U_{0}^{2}$. At the terminal of the linear region, $x / h=3$, peaks are observed at approximately 250,480 and $960 \mathrm{~Hz}$ in the power spectrum. These frequencies correspond to the subharmonic, fundamental and harmonic frequencies, respectively. That is, the subharmonic and harmonic waves exist at the end of the linear region. This fact is also recognized in Fig. 4.

Downstream, at $x / h=5$, periodic fluctuations also remain in the velocity signal. In the power spectrum density, besides an acute peak at approximately $200 \mathrm{~Hz}$, a wide bulge appears at approximately $100 \mathrm{~Hz}$. The sequential process of the vortex merging introduced by Hsiao-Huang ${ }^{(8)}$ starts from the merging of the fundamental wave, $f_{0}$, and an appearance of the subharmonic wave, $f_{0} / 2$. Then, after the saturation of $f_{0} / 2$, its harmonic wave, $f_{0}$, appears again from the modulation $\left(f_{0} / 2+f_{0} / 2\right)$. The same process continues after the an appearance of $f_{0} / 4$, until the disappearance of the potential core. They explained that this 
process was caused by the sideband instability based on the nonlinear interactions between the fundamental and subharmonic waves $^{(8)}$. The wide bulge of $100 \mathrm{~Hz}$ in the present experiment is estimated as the preferred frequency, $f_{p}$, because it remained in the end after the continuous merging of vortices. In fact, they reported that the preferred frequency, $f_{0} / 4$, was observed at the end of the potential core. In the present experiment, as mentioned before, the potential core disappeared at $x / h \simeq 6$, and the fundamental frequency, $f_{0}$, was approximately $480 \mathrm{~Hz}$. Thus, the results of the present experiment coincide with those of Hsiao-Huang.

At $x / h=7$, only the preferred frequency dominates. Though Hsiao-Huang measured up to the station where the preferred frequency remains, measurements in the present experiment were continued until reaching the station where the turbulent transition is completed. At $x / h=10$, the preferred frequency becomes unclear, and the power spectrum has almost changed to a continuous spectrum without specific peak frequencies. In the velocity signals, only irregular fluctuation is observed. At $x / h=14$, the turbulent transition has almost completed.

In the oscillating state, at $x / h=0.5$, the amplitude of a sinusoidal wave whose frequency is the same as the plate oscillation frequency, $5 \mathrm{~Hz}$, is large. A small amplitude oscillation whose frequency is approximately $400 \mathrm{~Hz}$ is superposed on it. At $x / h=1.5$, waves whose frequencies are the same as the plate oscillation frequency and its subharmonic frequency, can be seen. The fundamental, subharmonic and harmonic waves can also be observed well at $x / h=3$ in the stationary state. Their magnitudes are of the same order as the $5 \mathrm{~Hz}$-wave. At $x / h=4$, an irregular wave can be seen in the velocity signal. Here, the power spectrum density becomes smooth and the preferred frequency appears in a wide range at approximately $100 \mathrm{~Hz}$. The potential core disappears at $x / h \simeq 4$; this station almost coincides with the appearance of the preferred frequency. Under this oscillating state, the preferred frequency appears more in the upstream station than in the stationary state. At $x / h$ $=6$, only the velocity signal in the preferred frequency remains. At $x / h=8$, the preferred frequency in the velocity signal becomes unclear. The power spectral density has almost changed into a continuous spectrum. Thus, the station where the preferred frequency disappears is also more upstream than in the stationary state. At $x / h=10$, an irregular wave signal peculiar to the turbulent flows, dominates. Thus, in the natural transition process in the stationary state, fundamental wave appears first, then its harmonic and subharmonic waves appear, and the preferred frequency becomes dominant. In the end, the domination of irregular fluctuation is confirmed. In the oscillating state, each process in the natural transition process appears more in the upstream station by the large amplitude of the plate oscillation frequency. The turbulent transition is promoted by plate oscillation.

The transition from regular laminar flow to irregular turbulent flow and the reverse transition from turbulent to laminar flow are typical examples of important changes in fluid flows. How to express this transition process depends on how to represent the complexity or randomness of the flow. From an experimental point of view, an important condition of the measure is simply being able to measure with it. From the present author's experience, the intermittency factor is suitable for the normal transition ${ }^{(11) \sim(14)}$ and reverse transition ${ }^{(15)}$. To obtain the intermittency factor there, hot wire signals were distinguished between turbulent or non-turbulent. In gradual transition processes such as the present mixing layer, however, the intermittency factor is not always suitable.

In this sense, the randomness factor proposed by Sato et al. for free shear layer seems useful, though it seems not to have been applied for other purposes ${ }^{(3)(4)}$. Therefore, the randomness factor is applied to measure the transition process in the present mixing layer quantitatively. The randomness factor is defined as the ratio of an area in which a continuous spectral region of the power spectral density of the fluctuating velocity dominates. In the linear and nonlinear regions where band or line spectral regions are 
dominant, the continuous spectral region is less dominant. Therefore, the randomness factor is less than unity. On the other hand, after the completion of the transition, the line and band spectra disappear and only the continuous spectral dominates. Thus the randomness factor becomes unity. Details of the calculation method of the randomness factor will be explained in the appendix.

In Fig. 13 the streamwise variation of the randomness factor obtained from streamwise fluctuating velocity is shown. They are obtained at normal position, $y$, where the $u$ ' takes maximum at respective streamwise stations. Just after the nozzle exit in the stationary state, the flow is laminar and contains no periodic fluctuation. Thus, the randomness factor is almost at unity since the power spectrum is almost a continuous spectrum type. In the downstream, the periodic fluctuation appears and amplifies gradually, therefore, the randomness factor decreases. It takes minimum at around the terminal of the linear region, $x / h=3$. After that, it increases with the disappearance of the periodic fluctuation in the nonlinear region. At $x / h \simeq 16$, it becomes almost constant around unity. Also, with the velocity signal and power spectral in Figs. 11 and 12, the turbulent transition may be completed nearby.

In the oscillating state from just after the nozzle exit, the randomness factor is smaller than in the stationary state since the periodic fluctuation with plate oscillation frequency dominates. Also in this case, once it decreases and takes minimum, it increases as the stationary state. That is, it takes the same value at two different positions where the condition of the flow is different. The station where it takes the minimum value is more upstream than in the stationary state. The station where it becomes almost constant is around unity, $x / h \simeq 10$, which is more upstream than the stationary state. The turbulent transition in this case may be completed near this area.

In this way, for the process in which the band spectrum decreases and changes into the continuous spectrum, the randomness factor is regarded as valid, for it increases monotonically. In the early process, however, where the periodic fluctuation becomes dominant, Sato et al. did not report the fact that the randomness factor decreases ${ }^{(3)(4)}$. As opposed to the randomness factor, the intermittency factor which is often used in wall turbulence increases monotonically from zero to unity with the progress of turbulent transition. Therefore, the randomness factor cannot discriminate between the minute turbulence in the first laminar flow and the turbulence with vorticity after the completion of the transition.

To examine the $y$-distribution of the velocity fluctuation, contour maps of the randomness factor in a $x-y$ plane is shown in Fig. 14. Two white lines which show $U / U_{m}$ are 0.99 and 0.05 , and are also drawn in Fig. 4. The white region shows that the region has not been measured as Fig. 4. As shown in Fig. 14, the randomness factor is not constant in the $y$-direction.

In the linear region's stationary state, $x / h \leqq 3$, the randomness factor takes a minimum in $0.95 \leqq y /(h / 2) \leqq 1.0$, where the fluctuating velocity takes maximum. Thus, the periodic fluctuation contributes to the increase of velocity fluctuation. The minimum of the randomness factor in the whole region exists around the nozzle centerline, $y /(h / 2)=0$, in the

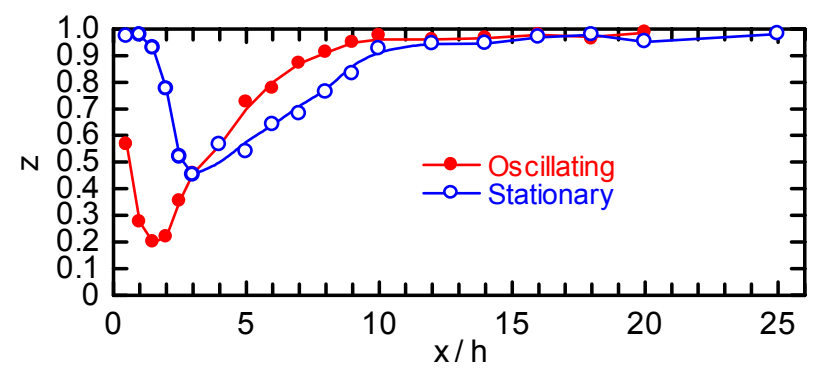

Fig. 13 Streamwise variation of randomness factor. 

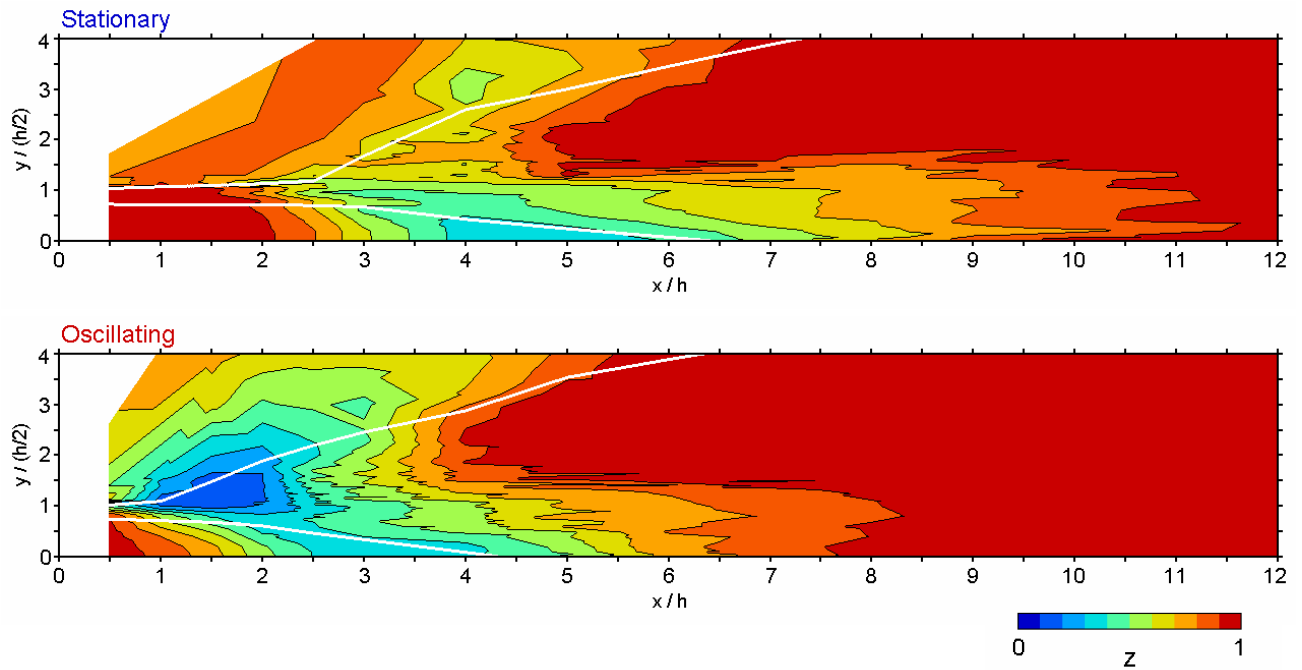

Fig. 14 Isocontour maps of randomness factor.

nonlinear region, $4 \leqq x / h \leqq 6$. In this region, the power spectra of the fundamental and subharmonic fluctuation take large values, as shown in Fig. 4. In the nonlinear region, the randomness factor does not become minimum where the fluctuating velocity takes maximum at $y /(h / 2) \simeq 1$. That is, in the nonlinear region the periodic fluctuation does not contribute to the increase of velocity fluctuation, as opposed to the linear region. Also on the centerline, the distribution of the randomness factor is shifted downstream from the maximum fluctuation points in Fig. 13.

In the linear region's oscillating state, $x / h \leqq 1.5$, the randomness factor takes minimum at $y /(h / 2) \simeq 0.95$, where the fluctuating velocity takes maximum as well as the stationary state. The minimum of the randomness factor in the whole region exists around the nozzle edge, at $y /(h / 2) \simeq 1$ and $1 \leqq x / h \leqq 2$. At the region of $2.5 \leqq x / h \leqq 4$, the randomness factor is smaller on the nozzle centerline than around the nozzle edge as well as at the region of $4 \leqq x / h \leqq 6$ in the stationary state. That is, at the station where the region of the potential core can be barely recognized, the randomness factor is smaller in the potential core than in other $y$-positions. From these randomness factors, it is considered that the turbulent transition in the oscillating state is promoted more than the stationary state.

Finally, the relationship between velocity fluctuation and periodic fluctuation is examined in detail. In the stationary state, as an example of a linear region, the station of $x / h$ $=2.5$ distributions in the $y$-direction of fluctuating velocity, the randomness factor and the power spectrum density at the fundamental and subharmonic frequencies are shown in Fig. $15(a)$. At $y /(h / 2) \simeq 0.95$ where the fluctuating velocity takes maximum, the randomness factor becomes minimum and the power spectra at fundamental and subharmonic frequencies are large. Nearer to the centerline, at $y /(h / 2)=0.7$ where the fluctuating velocity becomes slightly maximal, the randomness factor becomes slightly minimum and the power spectra at both frequencies are slightly enlarged. Therefore, in the linear region, the fluctuating velocity is large where the fundamental and subharmonic waves are dominant; therefore, the periodic fluctuation contributes to the rise of fluctuating velocity.

Farther downstream, in the nonlinear region where the periodic fluctuation disappears, as shown in Fig. 15(b), $x / h=6$, there is no maximum in the fluctuating velocity near the centerline, although the randomness factor becomes slightly smaller around $y /(h / 2)=1.0$. In this way, in the nonlinear region, the periodic fluctuation does not contribute to the rise in fluctuating velocity. The reason for the difference of the $y$-distribution between $x / h=2.5$ and 6 can be found in the difference of the $y$-position in the minimum randomness factor. The randomness factor takes minimum around the maximum position in the velocity fluctuation, $y /(h / 2) \simeq 0.95$ in the linear region. On the other hand, in the nonlinear region it 


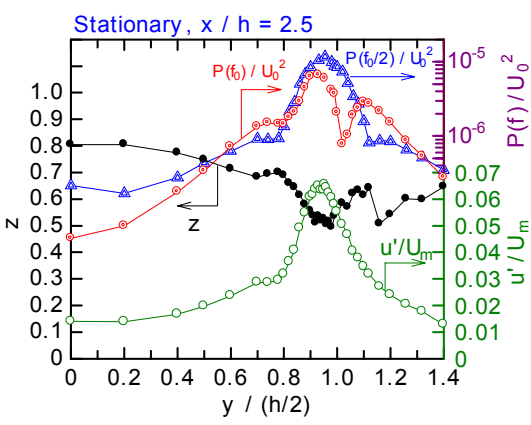

(a)

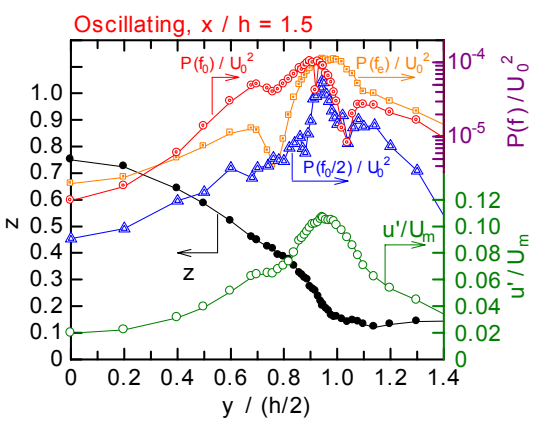

(c)

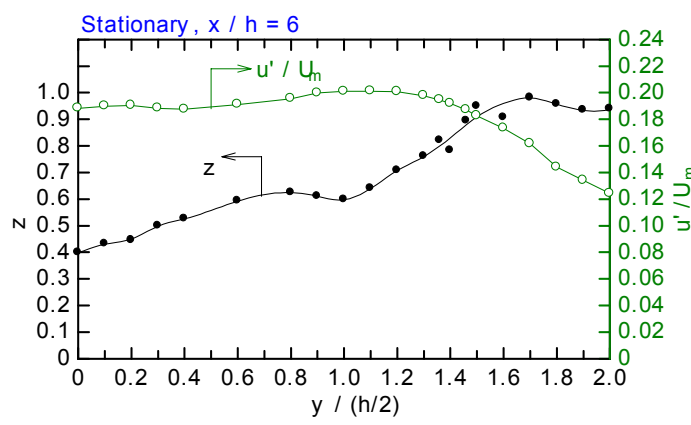

(b)

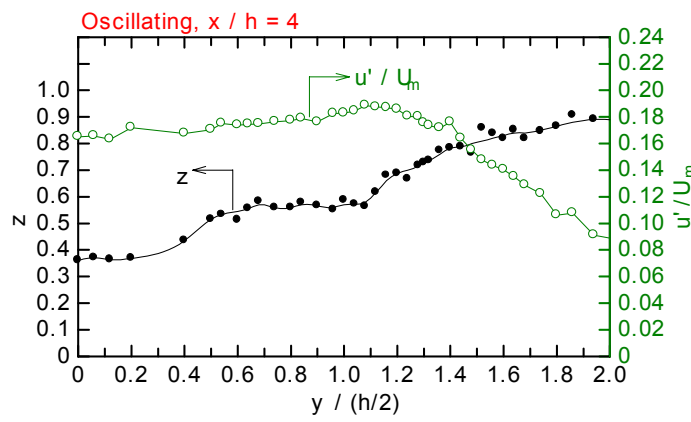

(d)

Fig. 15 Distributions of fluctuating velocity, randomness factor and power spectrum density.

takes minimum around the centerline, $y /(h / 2)=0$.

The oscillating state, at $x / h=1.5$ in the linear region is shown in Fig. 15(c), near the $y$-position of the maximum fluctuation, where the randomness factor is small and the power spectrum density at plate oscillation, fundamental, and subharmonic frequencies are maximum. Therefore, also in the oscillating state, periodic fluctuation contributes to the rise in fluctuating velocity.

In the nonlinear region at $x / h=4$, shown in Fig. $15(d)$, as well as the stationary state, Fig. 15(b), the randomness factor is not small around the maximum fluctuation position. The randomness factor becomes minimum at the centerline, and increases monotonically with the normal distance from the centerline.

\section{Conclusions}

Forced small-amplitude and low-frequency disturbances were locally added to a mixing layer formed at the exit of a two-dimensional nozzle. The laminar-turbulent transition in the mixing layer was investigated experimentally. The following conclusions were obtained within this Reynolds number.

(1) In the velocity signals in the oscillating state the periodic fluctuation with the oscillation frequency appeared at first. Next, the fundamental, subharmonic and harmonic waves were imposed on it. Then, the preferred frequency dominated. Finally, the turbulent flow with an irregular fluctuation dominated at all. The phenomenon which was observed in the natural transition process appeared more upstream, and the turbulent transition was completed earlier.

(2) In the early stage of the nonlinear region, due to the expansion of the mixing layer, the decrease of the velocity gradient, $-\partial U / \partial y$ and $-\partial V / \partial y$, lowered the production rate of the fluctuating velocity in the streamwise and normal directions and the Reynolds shear stress. Therefore, the growth rates of both fluctuating velocities weakened. The Reynolds shear stress once decreased, but increased again. The probabilities which positively contributed to the Reynolds shear stress decreased once and then increased again. 
(3) In the oscillating state, the Reynolds shear stress and its perturbation energy production rate just after the nozzle increased earlier than in the stationary state. Their maximums were larger than in the stationary state. Their effect, however, did not continue long due to their low frequency and small amplitude.

(4) As a quantitative measure of the turbulent transition, the randomness factor which has been proposed by Sato et al. was applied to the present mixing layer. In the process in which the periodic fluctuation changes into an irregular one, it increased monotonically, thus showing validity as a quantitative measure. On the other hand, upstream where laminar flow changes to the periodic fluctuating flow, the randomness factor decreased monotonically. In this way, it has a disadvantage in that it takes the same value at two stations where the flow conditions differ.

\section{Acknowledgments}

The authors deeply appreciate the kind instruction and constant encouragement of Prof. I. Nakamura of Nagoya University. The generous assistance of Messrs N. Hamada and T. Yasunobu of the University of Tokushima is also gratefully acknowledged.

\section{Appendix: How to estimate the randomness factor}

Here, the method for estimating the randomness factor is explained. Both axes in the schematic diagrams, Fig. 16, are drawn linearly.

(1) First, the original power spectrum density (Fig. 16(a)) is moving averaged with 5 points. The averaged spectrum is indicated in Fig. 16(b) with a blue line.

(2) If the averaged spectrum increases monotonically with the frequency, the spectrum is changed to be constant within the range. Then the "pseudo-continuous spectrum" without peaks is obtained, and expressed with the green line in Fig. 16(b).

(3) The threshold level is obtained as the pseudo-continuous spectrum times 0.2 (the red line in Fig. 16(b)). The value 0.2 has been selected from the visual check of a comparison between the original spectrum and the final randomness factor.

(4) The difference between the moving-averaged spectrum (blue line) and the pseudo-continuous spectrum (green line) in Fig. $16(b)$ is set to the discrimination function (blue line in Fig. 16(c)). In Fig. 16(c); the threshold level is also shown with a red line.

(5) The discrimination function is compared with the threshold level. If it is larger than the threshold level, the spectrum is regarded as a band spectrum region. The continuous spectrum is obtained from the original spectrum subtracted by the band spectrum and is shown in Fig. $16(d)$. As can be easily recognized, regions which contain peaks in the original spectrum have been removed.

(6) The randomness factor is the ratio of the area of the continuous spectrum, Fig. 16(d), to that of the original spectrum, Fig. 16(a).

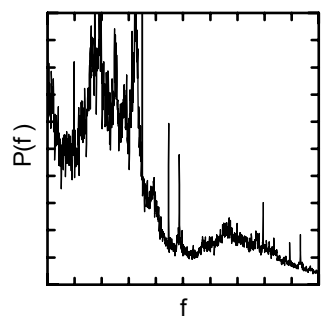

(a)

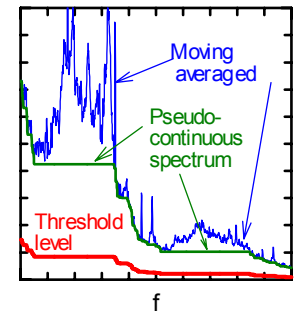

(b)

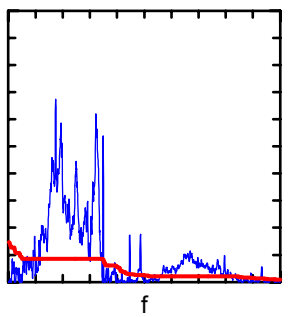

(c)

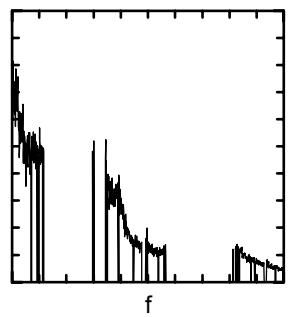

(d)

Fig. 16 Schematic diagrams for estimating randomness factor. 


\section{References}

(1) Durbin, P., and Wu,. X., Transition Beneath Vortical Disturbances, Annual Review of Fluid Mechanics, Vol.39 (2007), pp. 107-128.

(2) Sato, H., Laminar-Turbulent Transition in Free Shear Flow, in Tani, I. ed., Progress in Fluid Mechanics -Turbulent Flow- (1980), pp. 50, Maruzen (in Japanese).

(3) Ibid., p.75 (in Japanese).

(4) Sato, H., and Saito, H., Fine-Structure of Energy Spectra of Velocity Fluctuations in the Transition Region of a Two-Dimensional Wake, Journal of Fluid Mechanics, Vol. 67, Part 3 (1975), pp. 539-559.

(5) Ichimiya, M., Kato, T., and Morimoto, T., Effect of Local Periodic Disturbance on Mixing Layer at Exit of Two-Dimensional Jet, Journal of Fluid Science and Technology, Vol. 6, No. 6 (2011), pp. 887-901.

(6) Tatsumi, T., and Gotoh, K., Flow Stability Theory (1976), p.116, Sangyo Tosho Ltd. (in Japanese).

(7) Sato, H., Further Investigation on the Transition of Two-Dimensional Separated Layer at Subsonic Speeds, Journal of Physical Society of Japan, Vol. 14, No. 12 (1959), pp. 1797-1810.

(8) Hsiao, F. -B., and Huang, J. -M., Near-Field Flow Structures and Sideband Instabilities of an Initially Laminar Plane Jet, Experiments in Fluids, Vol. 9, No. 1-2 (1990), pp. 2-12.

(9) Sato, H., The Stability and Transition of a Two-Dimensional Jet, Journal of Fluid Mechanics, Vol. 7 (1960), pp. 53-80.

(10) Rotta, J. C., Turbulent Boundary Layers in Incompressible Flow, Ferri, A., Kuchemann, D. and Sterne, L.H.G. ed., Progress in Aeronautical Sciences, (1962), pp. 44, Pergamon Press.

(11) Ichimiya, M., Nakase, Y., Fukutomi, J., Yamana, K., and Fukunaga, M., Structure of a Turbulence Wedge Developed from a Single Roughness Element on a Flat Plate, Transactions of the Japan Society of Mechanical Engineers, Vol. 59, No. 557 (1993), pp. 129-136 (in Japanese).

(12) Ichimiya, M., Nakase, Y., Fukutomi, J., Kondou, S., and Tsutsui, I., Properties of a Turbulence Wedge Which Develops from a Single Roughness Element in a Flat Plate Laminar Boundary Layer, Transactions of the Japan Society of Mechanical Engineers, Vol. 67, No. 660 (2001), pp. 1983-1989 (in Japanese).

(13) Ichimiya, M., Abe, T., Fukutomi, J., and Kondo, M., Laminar-Turbulent Transition of a Boundary Layer induced by a Single Roughness Element in an Inlet Region of a Circular Pipe Flow (Developing Process of a Stationary Turbulent Region), Transactions of the Japan Society of Mechanical Engineers, Vol. 73, No. 725 (2007), pp. 154-161 (in Japanese).

(14) Ichimiya, M., Fujimura, H., and Tamatani, J., Laminar-Turbulent Transition of an Inlet Boundary Layer in a Circular Pipe Induced by Periodic Ejection (Condition for Generating an Isolated Turbulent Patch), Journal of Fluid Science and Technology, Vol. 6, No. 6 (2011), pp. 902-915.

(15) Ichimiya, M., Nakamura, I., and Yamashita, S., Properties of a Relaminarizing Turbulent Boundary Layer Under a Favorable Pressure Gradient, Experimental Thermal and Fluid Science, Vol. 17, No. 1-2 (1998), pp. 37-48. 